\title{
HoNorm and Variance Constrained Controller Design for Perturbed Stochastic System via Variable Structure Control
}

Wen-Jer Chang

Associate Professor, Department of Marine Engineering and Technology National Taiwan Ocean University, Keelung 202, Taiwan, R.O.C

Koan-Yuh Chang

Graduate Student, Department of Electrical Engineering, National Central University, Chung Li 320, Taiwan, R.O.C.

Follow this and additional works at: https://jmstt.ntou.edu.tw/journal

Part of the Engineering Commons

\section{Recommended Citation}

Chang, Wen-Jer and Chang, Koan-Yuh (2009) "HœNorm and Variance Constrained Controller Design for Perturbed Stochastic System via Variable Structure Control," Journal of Marine Science and Technology. Vol. 7: Iss. 1, Article 4. DOI: $10.51400 / 2709-6998.2509$

Available at: https://jmstt.ntou.edu.tw/journal/vol7/iss1/4

This Research Article is brought to you for free and open access by Journal of Marine Science and Technology. It has been accepted for inclusion in Journal of Marine Science and Technology by an authorized editor of Journal of Marine Science and Technology. 


\section{$\mathrm{H} \infty$ Norm and Variance Constrained Controller Design for Perturbed Stochastic System via Variable Structure Control}

\section{Acknowledgements}

The authors would like to thank two anonymous referees for their constructive comments and suggestions which have made substantial improvements on this paper. Besides, this work was supported by the National Science Council of the Republic of China under Contract NSC88-2213-E-019-007. 


\title{
$H_{\infty}$ NORM AND VARIANCE CONSTRAINED CONTROLLER DESIGN FOR PERTURBED STOCHASTIC SYSTEMS VIA VARIABLE STRUCTURE CONTROL
}

\author{
Wen-Jer Chang* and Koan-Yuh Chang**
}

Keywords: Sliding mode control, Upper bound covariance control, $H_{\infty}$ norm constraints, Variance constraints.

\section{ABSTRACT}

Based on the concept of variable structure control, this paper investigates the upper bound covariance assignment with $H_{\infty}$ norm and variance constrained problem for perturbed stochastic systems. Because of the invariance property of variable structure systems, the matched perturbation of the system disappears on the sliding mode. With the aid of Ito-formula, the controller $u(t)$ is proposed. Combining the sliding phase and hitting phase of the system design, the control feedback gain matrix $G$ is derived to achieve upper bound covariance assignment with $H_{\infty}$ norm and variance constraints.

\section{INTRODUCTION}

As one knows that how certain control objectives, robust stability and noise attenuation, will be achieved if certain $H_{\infty}$ bounds are achieved (see [18]). Hence, there has been lots of papers for the feedback controller design with $H_{\infty}$ norm constraints (see [1], [17] and [29]). In practice, we always require to develop some ways for designing controllers to achieve multiobjectives. There are some papers (see [2] and [28]) discussing the $H_{\infty}$ norm and variance constrained problem simultaneously. However, the Riccati equation approach applied by [2]

Paper Received Jan. 13, 1999. Revised Apr. 23, 1999. Accepted May 26, 1999. Author for Correspondence: Wen-Jer Chang.

*Associate Professor, Department of Marine Engineering and Technology National Taiwan Ocean University, Keelung 202, Taiwan, R.O.C.

**Graduate Student, Department of Electrical Engineering, National Central University, Chung Li 320, Taiwan, R.O.C. and [28], which minimizes a scalar cost index, does not ensure to satisfy the individual variance constraints. A more straightforward methodology (namely, covariance control) for designing controllers to achieve variance constraints of individual states has been developed in [11, 12, 19] and [27]. In [19], the authors do not consider the presence of system perturbations, hence, the system may become unstable when it is suffered from perturbations. An improved control method, which we call upper bound covariance control (UBCC) here, for satisfying variance constraints with perturbations was proposed in [11, 12] and [27]. Using the direct UBCC approach, the state feedback gain designed in $[11,12]$ and [27] will become very large when the systems are suffering from large perturbations. It is well known that a too large feedback gain is not encouraged in practical application.

As above mentioned, it is hard to design the state feedback control for perturbed stochastic system by using UBCC method directly. Owing to the simple design, easy implementability and insensitivity to system perturbations, variable structure control (VSC) has become a successful synthesis method and has been applied to many complex systems (see [16, 20, 21, 23, 24] and [25]). The main advantage of VSC system is that the system dynamics in the sliding mode are invariant if parameter uncertainties and/or perturbations satisfy a certain matching condition. Using the concept of VSC, the authors have discussed the stochastic large-scale systems [6, 7] and stochastic model reference systems [8,9] and [26]. In [5], the authors have successfully extended the above approach to linear perturbed systems. Moreover, for a practical application, the authors have successfully extended this combined technique to deal with the perturbed ship steering yaw-motion systems [10].

In this paper, based on the VSC concept and with 
the aid of UBCC, an improved design method will be developed in this paper for achieving $H_{\infty}$ norm and individual variance constraints. Consequently, we will use VSC approach to show that if a proper switching function is chosen, the controlled system in the sliding mode is insensitive to the perturbation (i.e., the system has an ability to reject the perturbation) and can be represented as a nominal linear feedback form (i.e., the system can be simplified). Then, based on the UBCC approach, the $H_{\infty}$ norm and individual variance constraints can be achieved on the sliding mode. Therefore, our propose has the ability of perturbation rejection, noise attenuation and robust stability that are our major contributions. Note that the upper bound covariance control solutions presented in this paper are independent to the system perturbations. Hence, the present solution forms will be more concise and efficient than that in $[11,12]$ and [27].

This paper is organized as follows. Section 2 describes the system statement and problem formulation. In section 3 , the switching function and sliding phase for VSC method are presented. Moreover, the hitting phase property of controller $u(t)$ is discussed in section 4. In section 5 , the $H_{\infty}$ norm and variance constrained design problems will be considered and the necessary and sufficient conditions for the existence of control feedback gain matrix $G$ is stated. A numerical example is given to demonstrate the control effect of the present method in section 6. Finally, some conclusions are made in section 7 .

\section{SYSTEM DESCRIPTION AND PROBLEM FORMULATION}

Consider a linear time-invariant perturbed stochastic system established on a filtered probability space $\left(\Omega, \mathcal{F},\left(\mathcal{F}_{t}\right)_{t \in R^{+}}, \mathcal{P}\right)$ and the system is described as

$$
\begin{aligned}
& \dot{x}(t)=(A+\Delta A) x(t)+B u(t)+D w(t), \\
& y(t)=F x(t),
\end{aligned}
$$

where $x(t), B$, and $D \in R^{n} ; F \in R^{1 \times n} ; A$ and $\Delta A \in R^{n \times n}$; $u(t) \in R^{1} ; w(t) \in R^{1}$ is a white noise input. Here, the white noise $w(t)$ of (1) satisfies (2)

$$
E(w(t))=0, E(x(0) w(t))=0, E\left(w^{2}(t)\right)=1,
$$

where $x(0)$ denotes the initial state, $(\cdot)^{T}$ denotes the transposition of $(\cdot)$. Suppose that $(A, B)$ is a stabilizable pair, and the perturbation, $\Delta A x(t)$, satisfies the perfect matching condition, i.e.,

$$
\operatorname{rank}[B: \Delta A]=\operatorname{rank}[B] .
$$

Moreover, we also assumption that the range space of matrix $B$ intercepts the range space of matrix $D$ only at the origin. The goals of this paper are as follows: how to design the controller $u(t)$ for the system such that

(i) The individual steady state variance constraints are satisfied, i.e.,

$$
[X]_{i i}=\operatorname{Var}\left(x_{i}\right) \leq[\bar{X}]_{i i} \leq \sigma_{i}^{2}, \quad i=1,2, \ldots n,
$$

where $\operatorname{Var}\left(x_{i}(t)\right)$ and $\sigma_{i}$ denote the variance value and root mean square (RMS) constraints for the $i$-th state of the system, $[\bar{X}]_{i i}$ denotes $i$-th diagonal element of upper bound covariance matrix $\bar{X}$ and $[X]_{i i}$ denotes the $i$-th diagonal element of matrix $X$. Here, we define $X$ as follows .

$$
X=\lim _{t \rightarrow \infty} E\left(x(t) x^{T}(t)\right) .
$$

(ii) In the sliding mode, the $H_{\infty}$ norm of transfer function from $w(t)$ to $y(t)$ is less than a fixed scalar for system robust stability.

The goal (i) is called the UBCC problem and the goal (ii) can be seen as a $H_{\infty}$ norm constrained problem. The presence of $\triangle A x(t)$ will make the UBCC problem with state feedback control be much more difficult. In order to avoid the difficulty from $\Delta A x(t)$, the invariance property of VSC is a nice choice for us to handle the perturbed system. Consequently, the concept of VSC will be utilized to carry out the UBCC with $H_{\infty}$ norm and variance constrained problem in this paper.

\section{SLIDING PHASE OF THE SYSTEM}

First we define the switching function $S(t)$ corresponding to $x(t)$ as follows

$$
S(t)=C x(t)-\int_{0}^{t}(C A+C B G) x(\tau) d \tau
$$

where $C$ and $G \in R^{1 \times n}$ are constant matrices to be designed. $C$ is chosen such that $C B$ is nonsingular, $C D$ $=0$ and $G$ is the control feedback gain matrix to be determined so that the state covariance can fit the requirement in the sliding mode. The switching function in (6) is well defined for the solution $x(t)$ of the system (1) (see [13] and [22]).

In the sliding mode, the states satisfy the equation

$$
S(t)=C x(t)-\int_{0}^{t}(C A+C B G) x(\tau) d \tau=0
$$

Differentiating equation (7) with respect to time, we obtain

$$
\dot{S}(t)=C \dot{x}(t)-C A x(t)-C B G x(t)=0 .
$$


Substituting (1a) into (8), we can get the equivalent control as follows

$$
u_{e q}(t)=G x(t)-(C B)^{-1} C(\Delta A x(t)+D w(t))
$$

Therefore, the equivalent dynamics of (1) in the sliding mode, $S(t)=0$, can be obtained by substituting (9) into (1) as

$$
\begin{aligned}
& \dot{x}(t)=(A+B G) x(t)+\left(I-B(C B)^{-1} C\right)(\Delta A x(t)+D w(t)), \\
& (10 a) \\
& y(t)=F x(t),
\end{aligned}
$$

where $I$ denotes the identity matrix. The invariance property (see [16]) had been known to be insensitive to perturbation in the sliding mode and it exists because of the perfect matching condition (3). Therefore, the dynamics (10) is insensitive to the perturbation $\Delta A x(t)$ and is thus reduced to,

$$
\begin{aligned}
& \dot{x}(t)=(A+B G) x(t)+\widetilde{D} w(t), \\
& y(t)=F x(t),
\end{aligned}
$$

where $\widetilde{D}=\left(I-B(C B)^{-1} C\right) D$. If we choose $C D=0$ then $\widetilde{D}=D$. Therefore, (11) can be rewritten as follows

$$
\begin{aligned}
& \dot{x}(t)=(A+B G) x(t)+D w(t), \\
& y(t)=F x(t) .
\end{aligned}
$$

Then how to design a controller $u(t)$ to guarantee the existence of the sliding mode and to choose the control feedback gain matrix $G$ so that the specified upper bound covariance matrix can be achieved in the sliding mode are our next works.

\section{A CONTROLLER DESIGN FOR HITTING MOTION}

Now, this section tries to find a controller $u(t)$ such that the states of the system (1) can be driven onto the sliding surface. Let us define a Lyapunov function

$$
V(S(t))=S^{2}(t)
$$

The following theorems will be given.

\section{Theorem 1}

Consider the system (1) with the solution $x(t)$. If a Lyapunov function $V(S(t))$ is designed as (13) and white noise $w(t)$ with intensity 1 is satisfied as (2) and
$C D=0$ is chosen, then we have

$$
\frac{d}{d t} V(S(t))=2 S(t) \dot{S}(t)
$$

Proof:

Suppose that $x(t)$ is a solution of (1) then $x(t)$ is a semimartingale ([13] and [22]). Since $S(t)$ is defined as (6), then $S(t)$ is also a semimartingale. From (13), and by Ito-formula ([13] and [22]), we can obtain

$$
\frac{d}{d t} V(S(t))=2 S(t) \dot{S}(t)+\frac{d}{d t}\langle S, S\rangle_{t},
$$

where $\langle S, S\rangle_{t}$ is an operation called bracket of $S(t)$ ([13] and [22]). Then we have

$$
\begin{aligned}
& \langle S, S\rangle_{t}=\left\langle C x-\int_{0} C(A+B G) x(\tau) d \tau\right. \\
& \left.C x-\int_{0} C(A+B G) x(\tau) d \tau\right\rangle_{t} \\
& =\langle C x, C x\rangle_{t} \\
& =\langle C D \mathcal{B}, C D \mathcal{B}\rangle_{t} \\
& =(C D)^{2}\langle\mathcal{B}, \mathcal{B}\rangle_{t} \\
& =(C D)^{2} t \\
& =0(\text { since } C D=0) .
\end{aligned}
$$

where $\mathcal{B}$ is the Brownian motion, i.e., $\mathcal{B}(t)=\int_{0}^{t} w(\tau) d \tau$. Substituting (21) into (15), we obtain (14). The proof is completed.

\section{Remark 1}

The sliding mode dynamics of the system (12) is independent of the vector $C$. The choice of $C$ to satisfy $C D=0$ is very easy and does not affect the sliding phase of (12).

\section{Theorem 2}

For the system (1), if $C D=0$ and let the controller $u(t)$ be

$$
u(t)=G x(t)-(C B)^{-1}\left[k_{1}\|C|\||| x(t)\|+\alpha] \operatorname{sgn}(S(t)),\right.
$$

where $k_{1}>\|\Delta A\|, \alpha$ is an arbitrary positive number; $\| x$ $(t)\|,\| \Delta A \|$ and $\|C\|$ denote the 2 -norm of the vector $x(t)$ and the induced 2-norm of the matrices $\|\Delta A\|$ and $C$, 


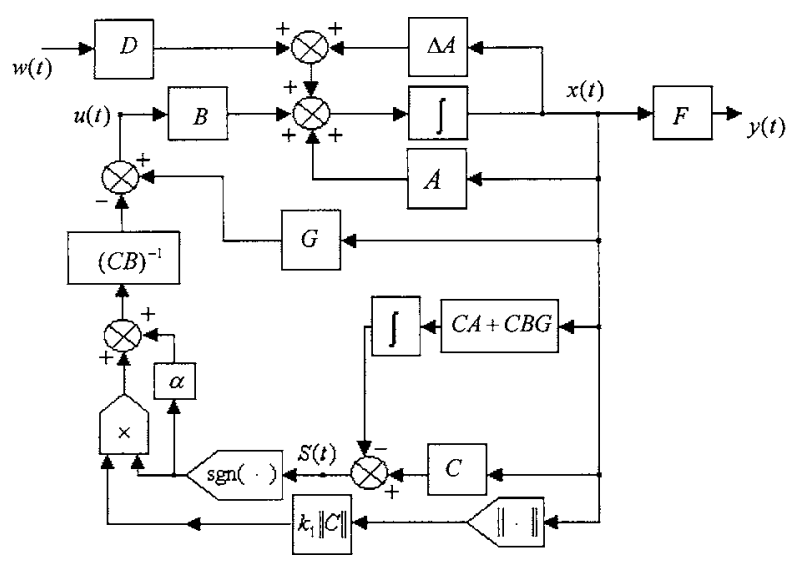

Fig. 1. The structure of the system (1) with the designed controller $u(t)$.

respectively. Then the state of system will converge to the sliding surface $S(t)=0$ with probability 1 .

\section{Proof:}

Differentiating (6) and choosing $C D=0$ and multiplying it by $S(t)$, we get

$$
S(t) \dot{S}(t)=S(t)[C B u(t)+C \Delta A x(t)-C B G x(t)]
$$

Substituting (22) into (23) and, then (23) becomes

$S(t) \dot{S}(t)=S(t) C \Delta A x(t)-k_{1}\|C\|\|x(t)\| \cdot|S(t)|-\alpha|S(t)|$.

Therefore, (14) becomes

$$
\begin{aligned}
\frac{d}{d t} V(S(t)) & =2 S(t) C \Delta A x(\mathrm{t})-2 k_{1}\|C\| \bullet\|x(t)\| \bullet|S(t)| \\
& -2 \alpha|S(t)| \\
& \leq 2|S(t) C \Delta A x(t)|-2 k_{1}\|C\| \bullet\|x(t)\| \bullet|S(\mathrm{t})| \\
& -2 \alpha|S(t)| \\
& \leq 2\|C\| \cdot\|\Delta A\| \bullet\|x(t)\| \cdot|S(t)|-2 k_{1}\|C\| \\
& \bullet\|x(t)\| \bullet|S(t)|-2 \alpha|S(t)| .
\end{aligned}
$$

If $k_{1}>\|\Delta A\|$ holds then (27) satisfies

$$
\frac{d}{d t} V(S(t)) \leq-2 \alpha|S(t)|<0
$$

That means the state $x(t)$ will converge to $S(t)=0$ with probability 1 . The proof is completed.

\section{Remark 2}

If $C D \neq 0,(14)$ becomes $\frac{d}{d t} V(S(t))=2 S(t) \dot{S}(t)+$ $(C D)^{2}$. That will make the control design of $u(t)$ be much more difficult.

\section{Remark 3}

For alleviating the phenomenon of chattering on the sliding mode, the boundary layer or sliding sector approach may be used to smooth out the control input (see [4], [14] and [15]). By using these methods, the robustness of control systems will be decreased and the system states will only reach to practical stability.

In order to express the overall scheme of the system (1) with the designed $u(t)$ (22), a block diagram of the system structure is shown in Fig. 1.

\section{THE CONTROL FEEDBACK GAIN MATRIX $G$ DESIGN WITH $H_{\infty}$ NORM AND VARIANCE CONSTRAINTS}

From the last two sections, the controller $u(t)$ in (22) forces the state to hit the sliding surface (7). According to the matching condition (3), the invariance property exists in the sliding mode and the sliding mode dynamics is (12). The left problems are that does the matrix $G$ exist and how to get it to achieve the goals (i) and (ii)?

Now, consider the system dynamics (12) in the sliding mode. The transfer function $H(p)$ from noise input $w(t)$ to output $y(t)$ is ,

$$
H(p) \triangleq F(p I-A-B G)^{-1} D,
$$

where $p$ is the Laplace operator. Therefore, the goal (ii) can be rewritten as (30)

$$
\|H(p)\|_{\infty} \leq \gamma
$$

for some prescribed positive constant $\gamma$.

For the moment, let us consider a useful lemma which was presented in the Reference [2].

\section{Lemma 1 [2]}

Consider the system (12). Let $G$ be given, $H(p) \in$ $R \mathcal{H}_{\infty}, \gamma>0$ and assume there exits a positive definite matrix $\bar{X}$ satisfying

$$
(A+B G) \bar{X}+\bar{X}(A+B G)^{T}+\gamma^{-2} \bar{X} \tilde{R} \bar{X}+D D^{T}=0,
$$

where $\tilde{R}=F^{T} F$, then

$$
\left(A+B G,\left[\gamma^{-2} \bar{X} \tilde{R} \bar{X}+D D^{T}\right]^{1 / 2}\right) \text { is stabilizable,(32) }
$$

if and only if $(A+B G)$ is asymptotically stable. In this case, 


$$
\|H(p)\|_{\infty} \leq \gamma
$$

and

$$
X \leq \bar{X}
$$

Proofs of Lemma 1 are found in [2]. Note that if the matrix $\bar{X}$ is positive definite, then the equation (32) is stabilizable. Hence, the purpose of this section may be restated as follows.

Given a positive scalar $\gamma$ and a symmetric positive definite matrix $\bar{X}$ which satisfies the constraints $[X]_{i i} \leq$ $[\bar{X}]_{i i} \leq \sigma_{i}^{2}$. Determine the control feedback gain matrix $G$ such that the equation (31) is satisfied for the specified $\gamma$ and $\bar{X}$.

In the following lemma, we will introduce a method to check whether the $H_{\infty}$ norm constraint (33) of the system (12) is satisfied. The proofs of Lemma 2 can be found in [3].

\section{Lemma 2 [3]}

Consider the system (12). There exists a positive scalar $\gamma$ to satisfy $\|H(p)\|_{\infty} \leq \gamma$, if and only if $M_{\gamma}$ has no eigenvalues on the imaginary axis, where

$$
M_{\gamma}=\left[\begin{array}{cc}
A+B G & \gamma^{-1} D D^{T} \\
-\gamma^{-1} F^{T} F & -(A+B G)^{T}
\end{array}\right] \text {. }
$$

It follows from Lemma 1 that the satisfaction of (31) leads to $(A+B G)$ is asymptotically stable; $H_{\infty}$ norm bound $\gamma$, and an upper bound covariance matrix $\bar{X}$ for the state covariance matrix $X$. Hence, in this section we will first derive the conditions and solutions for which (31) is satisfied. Then, the controller solutions $G$ will be characterized by the UBCC which is based on the approach of singular value decomposition and the theory of generalized inverse.

\section{Theorem 3}

Consider the system (12) Given an upper bound covariance matrix $\bar{X}=\bar{X}^{T}>0$, then there exists a control feedback gain matrix $G$ such that $\bar{X}$ solves (31), where

$$
G=B^{+}(Q-\bar{X})+\left(I-B^{+} B\right) Z
$$

in which $Z$ is an arbitrary matrix if and only if

$$
\left(I-B B^{+}\right)(Q-\bar{X})=0,
$$

and

$$
(A+Q) \bar{X}+\bar{X}(A+Q)^{T}+\bar{X}\left(\gamma^{-2} \tilde{R}-2 I\right) \bar{X}+D D^{T}=0
$$

for some $Q$, where $Q \in R^{n \times n}$ and $(\bullet)^{+}$denotes the MoorePenrose inverse of $(\bullet)$.

\section{Proof:}

Necessity : Suppose there exists a $G$ satisfying (31) for given $\bar{X}>0$. (31) can be rewritten as

$$
\begin{aligned}
& A \bar{X}+\bar{X} A^{T}-\overline{X X}-B G G^{T} B^{T}+\gamma^{-2} \bar{X} \tilde{R} \bar{X}+D D^{T}+Q Q^{T} \\
& \quad=0,
\end{aligned}
$$

where

$$
Q=B G+\bar{X}
$$

By assumption $G$ satisfying (39) exists. Hence, (39) has to be solvable for $G$, which is guaranteed if and only if

$$
\left(I-B B^{+}\right)(Q-\bar{X})=0
$$

The solution $G$ is given by

$$
G=B^{+}(Q-\bar{X})+\left(I-B^{+} B\right) Z,
$$

where $Z$ is arbitrary matrix. Substituting (41) into (38) yields

$$
(A+Q) \bar{X}+\bar{X}(A+Q)^{T}+\bar{X}\left(\gamma^{-2} \tilde{R}-2 I\right) \bar{X}+D D^{T}=0
$$

where we used the relation given by (40). This completes the necessity of the existence of $G$ satisfying (36) and (37).

Sufficiency : Now suppose $\bar{X}>0$ satisfies (36) and (37) for some $Q$. For suficiency, we will show that $G$ given by (35) solves (31). From (35) and (36),

$$
B G=B B^{+}(Q-\bar{X})=Q-\bar{X}
$$

Putting (43) into the left-hand side of (31) yields

$$
\begin{aligned}
& (A+B G) \bar{X}+\bar{X}(A+B G)^{T}+\gamma^{-2} \bar{X} \tilde{R} \bar{X}+D D^{T} \\
& =(A+Q) \bar{X}+\bar{X}(A+Q)^{T}+\bar{X}\left(\gamma^{-2} \tilde{R}-2 I\right) \bar{X}+D D^{T}
\end{aligned}
$$

But since (42) holds, the right-hand side of (44) is zero. Hence, (31) holds for $G$ given by (35). This completes the suffiency of the existence of $G$ satisfying (36) and (37). 
When the system (12) satisfies the necessary and sufficient conditions of the Theorem 3 , then there exits a control feedback gain matrix $G$ such that the specified upper bound covariance matrix $\bar{X}$ will be achieved for the equation (31).

Let us summarize the above design procedure to be the following steps.

Initial status: The system (1) is given with certain assumptions in section 2. An upper bound covariance matrix $\bar{X}$ is pre-specified.

Step 1: Choose $C$ to satisfy $C D=0$ and $C B \neq 0$.

Step 2: Find some $Q$ to satisfy (36) and (37), then get $G$ from (35).

Step 3: Get the switching function $S(t)$ as (6).

Step 4: The controller (22) is obtained.

\section{A NUMERICAL EXAMPLE}

In this section, we consider a perturbed system as (1) and it's perturbation satisfies the matching condition. We will use our proposed method and the method of [27], respectively, to solve the problem and also make a comparison between them. Now, consider a linear timeinvariant stochastic system (1) with the system parameters

$$
\begin{aligned}
& A=\left[\begin{array}{cc}
0 & 1 \\
-2 & -4
\end{array}\right], B=\left[\begin{array}{c}
1 \\
-1
\end{array}\right], D=\left[\begin{array}{l}
0 \\
1
\end{array}\right] \\
& \Delta A=10 \delta\left[\begin{array}{cc}
1 & -0.8 \\
-1 & 0.8
\end{array}\right], F=\left[\begin{array}{ll}
1 & 1
\end{array}\right],
\end{aligned}
$$

where $\delta \in[0,1]$ is an uncertain parameter. Here, we suppose that the system is driven by initial states $\mathrm{x}(0)=$ $\left[\begin{array}{ll}x_{1}(0) & x_{2}(0)\end{array}\right]^{T}=\left[\begin{array}{ll}7 & 7\end{array}\right]^{T}$ and white noise $w(t)$ which satisfies (2) with identiy covariance. The design goals are to find the controller $u(t)$ such that the steady state of the system satisfies the following requirements.

$$
\begin{aligned}
& \|H(p)\|_{\infty}<1, \\
& \operatorname{Var}\left(x_{1}(t)\right)<1.5, \operatorname{Var}\left(x_{2}(t)\right)<3 .
\end{aligned}
$$

\section{Case (i): Using the present approach}

If we pre-specify the upper bound covariance matrix $\bar{X}=\left[\begin{array}{ll}1 & 1 \\ 1 & 2\end{array}\right]$, which has diagonal elements satisfying the performance constraints (47). Then, the proposed design procedure may be obtained as follows.

Step 1: Choosing $C=\left[\begin{array}{ll}1 & 0\end{array}\right]$ such that $C D=0$. Clearly, the perturbation $\Delta A x(t)$ disappears on the slid- ing mode.

Step 2: Substituting $\bar{X}$ into Theorem 3, the conditions (36)-(37) are satisfied with $Q=\left[\begin{array}{cc}0 & -1 \\ 2 & 4\end{array}\right]$.

Hence, from (35) we may obtain the control feedback gain matrix as follows.

$$
G=\left[\begin{array}{ll}
-1 & -2
\end{array}\right]
$$

Step 3: From (6), the switching function has the following form.

$$
S(t)=\left[\begin{array}{ll}
1 & 0
\end{array}\right] x(t)-\int_{0}^{t}[-1-1] x(\tau) d \tau
$$

Step 4: A controller $u(t)$ is obtained by choosing $k_{1}=$ 18.5 and $\alpha=1$ from Theorem 2. Then, the controller $u(t)$ becomes

$$
u(t)=[-1-2] x(t)-[18.5\|x(t)\|+1] \operatorname{sgn}(S(t))
$$

For the comparison with UBCC technique in [27], Table 1 shows the results of control effects (the individual variances of state $x_{1}(t)$ and $x_{2}(t)$ and control feedback gain $G$ ) with respect to uncertain parameter $\delta$ ( $\delta=0.2, \delta=0.4, \delta=0.6, \delta=0.8$, and $\delta=1$ ).

From the above design procedure, we can conclude that the upper bound covariance matrix $\bar{X}$ will be achieved if the system is driven by controller (50) to the sliding mode. In the simulation of case (i), $\delta$ is chosen as a random number between 0 and 1 . The time response of states $x_{1}(t)$ and $x_{2}(t)$ are given in Fig. 2 and Fig. 3, respectively. The time response of $S(t)$ and $u(t)$ are shown in Fig. 4 and Fig. 5, respectively. For stability, the phase plane of $\left(x_{1}(t), x_{2}(t)\right)$ is shown in Fig. 6. It is easy to check that the matrix $M_{\gamma}$ which defined in Lemma 2 has no imaginary eigenvalues, hence the $H_{\infty}$ norm constraint (46) is satisfied. Moreover, the variances of $x_{1}(t)$ and $x_{2}(t)$ are 0.0521 and 0.1628 , respectively. Therefore, the individual variance constraints (47) are also achieved.

\section{Case (ii): Using the UBCC technique developed in [27]}

Pre-specifying the upper bound covariance matrix $\bar{X}=\left[\begin{array}{ll}1 & 1 \\ 1 & 2\end{array}\right]$ and using the UBCC approach of [27], we can obtain the feedback controller $G$ respect to $\Delta A$. Table 2 shows the individual variances of state $x_{1}(t)$ and $x_{2}(t)$ and control feedback $G$ due to different uncertain parameters $\delta$.

In Case (i) we can see that the feedback gain $G$ is independent of perturbation. Therefore, if $\Delta A$ is increased then $G$ is still a constant gain. However, the 
Table 1. State variances and control feedback gain using the present method

\begin{tabular}{cccccc}
\hline & $\delta=0.2$ & $\delta=0.4$ & $\delta=0.6$ & $\delta=0.8$ & $\delta=1$ \\
\hline $\operatorname{Var}\left(x_{1}(\mathrm{t})\right)$ & 0.0573 & 0.0542 & 0.0493 & 0.0539 & 0.0500 \\
$\operatorname{Var}\left(x_{2}(\mathrm{t})\right)$ & 0.1539 & 0.1586 & 0.1684 & 0.1620 & 0.1686 \\
$\mathrm{G}$ & {$[-1-2]$} & {$[-1-2]$} & {$[-1-2]$} & {$[-1-2]$} & {$[-1-2]$} \\
\hline
\end{tabular}

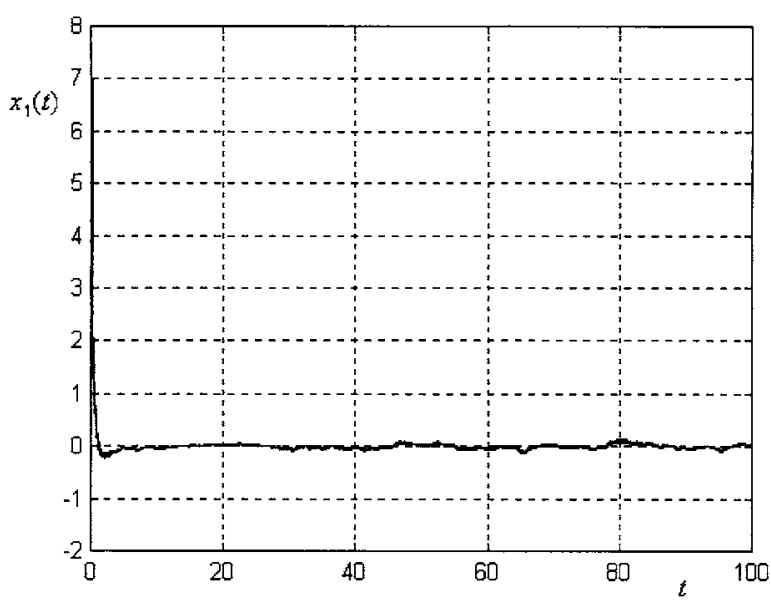

Fig. 2. Time response of state $x_{1}(t)$.

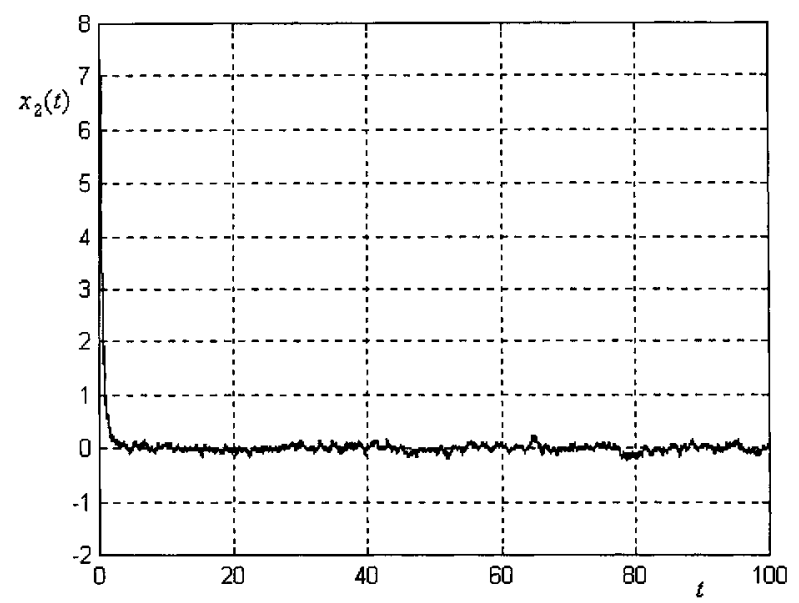

Fig. 3. Time response of state $x_{2}(t)$.

UBCC feedback gain $G$ will become large if $\Delta A$ increases in Case (ii).

\section{CONCLUSIONS}

This paper has provided a method to design a controller $u(t)$ to achieve the UBCC with $H_{\infty}$ norm and variance constraints. Based on the concept of VSC, we can obtain the linear closed-loop system without

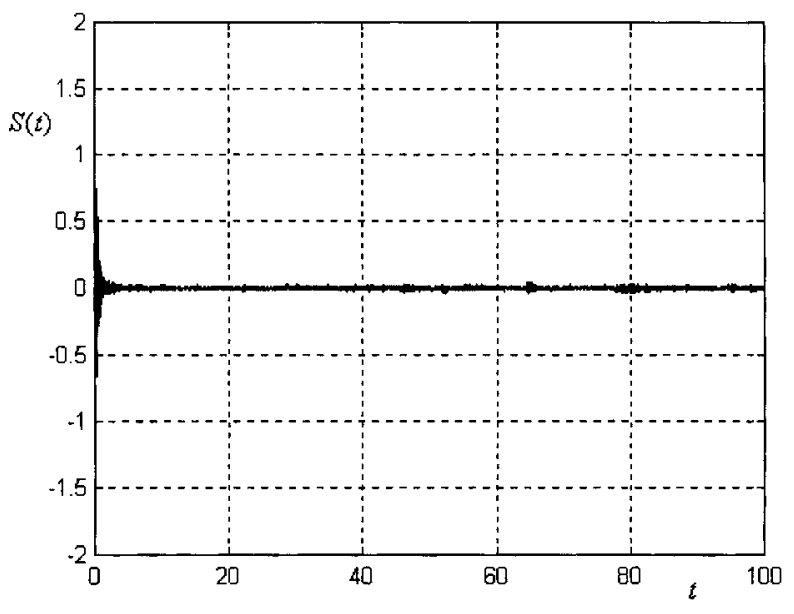

Fig. 4. Time response of $S(t)$.

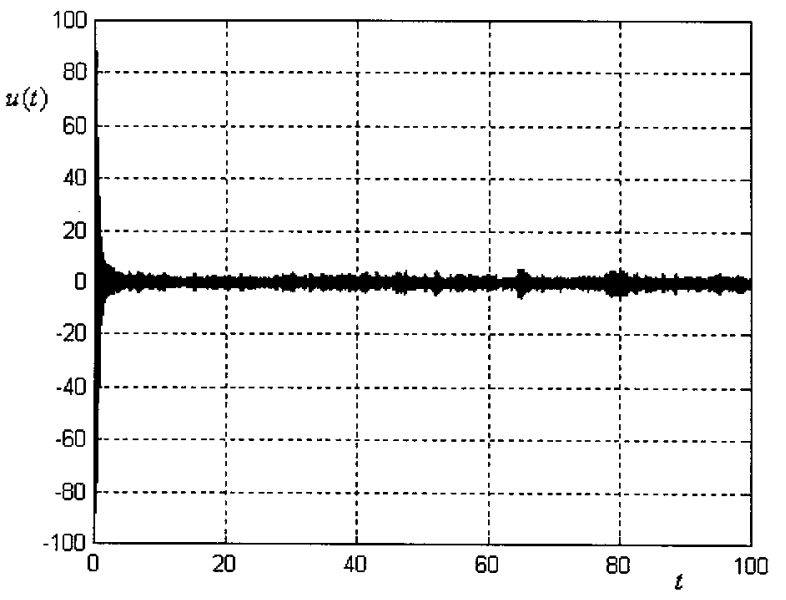

Fig. 5. Time response of $u(t)$.

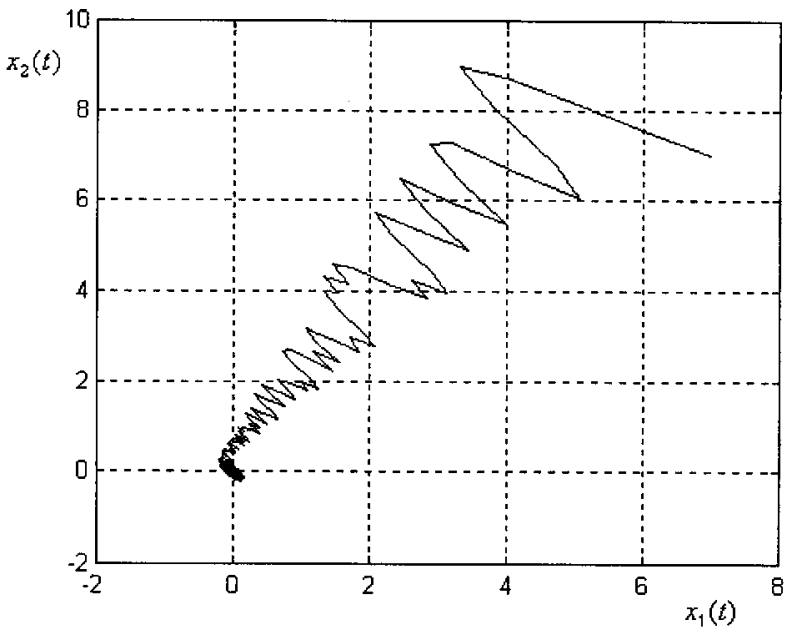

Fig. 6. The phase plane of $\left(x_{1}(t), x_{2}(t)\right)$. 
Table 2. State variances and control feedback gain using UBCC method of [27]

\begin{tabular}{cccccc}
\hline & $\delta=0.2$ & $\delta=0.4$ & $\delta=0.6$ & $\delta=0.8$ & $\delta=1$ \\
\hline $\operatorname{Var}\left(x_{1}(\mathrm{t})\right)$ & 0.1629 & 0.0775 & 0.0683 & 0.0657 & 0.0645 \\
$\operatorname{Var}\left(x_{2}(\mathrm{t})\right)$ & 0.0672 & 0.1223 & 0.1323 & 0.1353 & 0.1367 \\
$\mathrm{G}$ & {$\left[\begin{array}{c}-4.1168 \\
1.6420\end{array}\right]^{T}$} & {$\left[\begin{array}{c}-19.4672 \\
11.8756\end{array}\right]^{T}$} & {$\left[\begin{array}{c}-45.0512 \\
28.9316\end{array}\right]^{T}$} & {$\left[\begin{array}{c}-80.8688 \\
52.8100\end{array}\right]^{T}$} & {$\left[\begin{array}{c}-129.920 \\
83.5108\end{array}\right]^{T}$} \\
\hline
\end{tabular}

perturbation. Since the utilization of VSC, the designed control feedback gain matrix $G$ not only achieve the $H_{\infty}$ norm and variance constraints but also determine the sliding surface. Furthermore, in order to satisfy the hitting motion to the sliding surface, Ito-formula has been used to solve the effect of white noise $w(t)$. This paper is a new trial to combine VSC and UBCC for achieving $H_{\infty}$ norm and individual variance constraints for perturbed linear stochastic systems.

\section{ACKNOWLEDGEMENTS}

The authors would like to thank two anonymous referees for their constructive comments and suggestions which have made substantial improvements on this paper. Besides, this work was supported by the National Science Council of the Republic of China under Contract NSC88-2213-E-019-007.

\section{REFERENCES}

1. Ball, J.A. and Cohen, N., "Sensitivity Minimization in an $H_{\infty}$ Norm: Parametrization of All Suboptimal Solutions," International Journal of Control, Vol. 46, No. 4, pp. 785-816 (1987).

2. Bernstein, D.S. and Haddad, W.M., "LQG Control with an $H_{\infty}$ Performance Bound : A Riccati Equation Approach," IEEE Transactions on Automatic Control, Vol. 34, No. 2, pp. 293-305 (1989).

3. Boyd, S.P. and Barratt, C.H., 1991, Linear Controller Design, Limits of Performance, Prentice-Hall, Inc., New Jersey (1991).

4. Burton, J.A. and A.S.I. Zinober, "Continuous Approximation of Variable Structure Control," International Journal of Systems and Science, Vol. 17, No. 6, pp. 875885 (1986).

5. Chang, K.Y. and Wang, W.J., "Robust Covariance Control for Perturbed Stochastic Multivariable System via Variable Structure Control," Systems \& Control Letters, to appear (1999).

6. Chang, K.Y. and Wang, W.J., "Local State Covariance Assignment for Stochastic Large-scale Systems," ASME Journal of Dynamic Systems, Measurement, and Control, Vol. 121, No. 1, pp.139-142 (1999).
7. Chang, K.Y. and Wang, W.J., " $H_{\infty}$ Norm Constraint and Variance Control for Stochastic Uncertain Large-scale Systems via Sliding Mode Concept," IEEE Transactions on Circuits and Systems -I Fundamental Theory and Applications, to appear (1999).

8. Chang, K.Y. and Wang, W.J., "Covariance Assignment for Stochastic Model Reference Systems via Sliding Mode Control Concept," Proceedings of the American Control Conference, Philadelphia, PA, USA, June, pp. 289-290 (1998).

9. Chang, W.J. and Chang, K.Y., " $H_{\infty}$ Norm and Variance Constrained Controller Design for Stochastic Model Reference Systems via Sliding Mode Control Concept," Proceedings of the American Control Conference, Hyatt Regency San Diego, California, USA, June, pp. 28032807 (1999).

10. Chang, W.J. and Chang, K.Y., "Performance Constrained Variable Structure Control for Ship Yaw-motion Systems with Perturbations," Proceedings of the IFAC Conference on Control Applications in Marine Systems (CAMS'98), Fukuoka, Japan, October, pp. 23-28 (1998).

11. Chang, W.J. and Chung, H.Y., "Upper Bound Covariance Control of Discrete Perturbed Systems," Systems \& Control Letters, Vol. 19, No. 6, pp. 493-498 (1992).

12. Chung, H.Y. and Chang, W.J., "Covariance Control with Variance Constraints for Continuous Perturbed Stochastic Systems," Systems \& Control Letters, Vol. 19, No. 5, pp. 413-417 (1992).

13. Dellacherie, C. and Meyer, P.A., Probabilities and Potential B, North-Holland, Amsterdam (1982).

14. Dorling, C.M. and A.S.I. Zinober, "Two Approaches to Hyperplane Design in Multivariable Structure Control Systems," International Journal of Control, Vol. 44, No. 1, pp. 65-82 (1986).

15. Dorling, C.M. and A.S.I. Zinober, "Robust Hyperplane Design in Multivariable Variable Structure Control Systems," International Journal of Control, Vol. 48, No. 5, pp. 2043-2054 (1988).

16. Drazenovic, B., "The Invariance Conditions in Variable Structure Systems," Automatica, Vol. 5, No. 2, pp. 287295 (1969).

17. Francis, B.A. and Doyle, J.C., "Linear Control Theory with an $H_{\infty}$ Optimality Criterion," SIAM Journal on Control Optimization, Vol. 25, pp. 815-844 (1987). 
18. Francis, B.A., A Course in $H_{\infty}$ Control Theory, SpringerVerlag, New York (1987).

19. Hotz, A. and Skeleton, R.E., "Covariance Control Theory," International Journal of Control, Vol. 46, No. 1, pp. 13-32 (1987).

20. Hung, J.Y., Gao, W.B. and Hung, J.C., "Variable Structure Control: A Survey," IEEE Transactions on Industrial Electronics, Vol. 40, No. 1, pp. 2-22 (1993).

21. Lee, J.L. and Wang, W.J., "Robust Decentralized Stabilization via Sliding Mode Control," Control-Theory and Advanced Technology, Vol. 9, No. 3, pp. 721-732 (1993).

22. Revuz, D. and Yor, M., Continuous Martingales and Brownian Motion, Springer-Verlag, Berlin Heidelberg (1991).

23. Utkin, V.I., "Variable Structure Control Systems with Sliding Modes," IEEE Transactions on Automatic Control, Vol. 22, No. 2, pp. 212-222 (1977).

24. Utkin, V.I., "Discontinuous Control Systems: State of the Art in Theory and Applications," Proceegings of 10th IFAC Triennial World Congress, Munich, Vol. 1, pp. 25-44 (1987).

25. Wang, W.J. and Lee, J.L., "Hitting Time Reduction and Chattering Attenuation in Multi-input Variable Structure Systems," Control-Theory and Advanced Technology, Vol. 9, No. 2, pp. 491-500 (1993).

26. Wang, W.J. and Chang, K.Y., "Variable Structure Based Covariance Assignment for Stochastic Multivariable Model Reference Systems," Automatica, to appear (1999).

27. Xu, J.H., Skelton, R.E. and Zhu, G., "Upper and Lower Covariance Bounds for Perturbed Linear Systems," IEEE Transactions on Automatic Control, Vol. 35, No. 8, pp. 944-948 (1990).

28. Yeh, H.H., Banda, S.S., Heise, S.A. and Bartlett, A.C., "Robust Control Design with Real-parameter Uncertainty and Unmodeled Dynamics," Journal of Guidance,
Control, and Dynamics, Vol. 13, No. 6, pp. 1117-1125 (1990).

29. Zames, G., "Feedback and Optimal Sensitivity: Model Reference Transformations, Multiplitycative Seminorm and Approximate Inverse," IEEE Transactions on Automatic Control, Vol. 26, No. 2, pp. 301-320 (1981).

\section{使用可變結構控制法設計滿足擾動 隨機系統 $H_{\infty}$ 模限制與方差限制之控 制器}

張文哲

國立台灣海洋大學輪機工程技術系

$$
\text { 張 寬 裕 }
$$

國立中央大學電機工程學系

摘 要

以可變結構控制理論爲基礎，本篇論文將透過 上界協方差的指定來達到滿足擾動隨機系統 $H_{\infty}$ 模限 制與方差限制的目的。由於可變結構系統的特性, 符 合匹配條件的擾動項, 將在滑動模式下消失, 在此情 形下結合Ito公式, 則控制器便可被設計出。另外, 我 們將説明如何應用可變結構系統的控制技巧, 以便推 導出符合指定之上界協方差矩陣的控制回授增益, 使 得閉迴路系統同時滿足 $H_{\infty}$ 模限制與方差限制。 